\title{
Recognising covert disease in women with chronic vulval symptoms attending an STD clinic: value of detailed examination including colposcopy
}

\author{
M A BYRNE, ${ }^{*} \dagger$ M M WALKER, J LEONARD,§ D PRYCE, * D TAYLOR-ROBINSON*† \\ From the *Jefferiss Research Wing of the Praed Street Clinic, the Departments of $\ddagger$ Histopathology and \\ $\S$ Dermatology, St Mary's Hospital, London, and the †Division of Sexually Transmitted Diseases, Clinical \\ Research Centre, Harrow, Middlesex
}

SUMmaRY A vulval colposcopy clinic was established in the sexually transmitted diseases (STD) clinic of St Mary's Hospital, London, to investigate patients with chronic symptoms (mean duration $3 \frac{1}{2}$ years). Fifty patients with a mean age of 33 were studied for 12 months, and a specific diagnosis was established for many of them. Forty complained of pruritus vulvae, 33 of dyspareunia, and 30 of vulvodynia. One or more macroscopic abnormality was seen in 29 of the women, the most common being erythema, papules, plaques, and fissures. Colposcopic abnormalities, however, were identified in 45 of the women, the most common being acetowhite epithelium in a flat, papillary, or cobblestone pattern. Biopsy specimens were obtained from 33 of the patients, and all had abnormal histological features. Those of human papillomavirus (HPV) infection were the most common, and were found in 27 specimens; evidence of vulval intraepithelial neoplasia was seen in seven and that of dermatoses in 10. Further work is required, however, before concluding that HPV was necessarily responsible for the chronic symptoms.

In many sexually transmitted diseases (STD) clinics there is a subset of patients with chronic, often longstanding, vulval symptoms for which no clear cause can be found by routine investigation. These women usually attend a clinic on multiple occasions and are seen by many different doctors in their quest for relief of their symptoms. A range of diagnoses from chronic non-specific irritation to psychosomatic disease is often put forward. To ascertain whether an underlying physical cause exists in such women we investigated a group in detail, undertaking colposcopic examination of the vulva. We report our observations here.

\section{Patients and methods}

A specific clinic, the vulval colposcopy clinic, was instigated to investigate women referred from the general STD clinic because they had one or more of the following symptoms: pruritus vulvae, dyspareunia, or

Address for reprints: Dr D Taylor-Robinson, Division of Sexually Transmitted Diseases, Clinical Research Centre, Watford Road, Harrow, Middlesex HA1 3UJ

Accepted for publication 22 August 1988 vulvodynia. Vulvodynia was defined, according to the recommendation of the International Society for the Study of Vulvar Disease, as chronic vulval discomfort especially characterised by burning. ${ }^{1}$ This was differentiated from pruritus vulvae, which is associated with chronic scratching. Dyspareunia was superficial rather than deep in character, and ranged from occasional mild discomfort to complete preclusion of sexual intercourse. We saw 50 patients (aged 19 to 56 (mean 33) years) in 12 months. Each was allotted a one hour appointment.

Assessment of each patient included taking a detailed medical history and making a general physical examination. In particular, the external genitalia were inspected carefully and then the entire lower genital tract was examined using a Zeiss colposcope. The vulva and cervix were evaluated both before and two to four minutes after the application of $5 \%$ acetic acid. All abnormalities identified in this way were documented diagrammatically. Punch biopsy specimens 4 $\mathrm{mm}$ in diameter were obtained from abnormal areas in a proportion of patients only, as some were unwilling to undergo biopsy. Specimens were placed in $10 \%$ formol saline, fixed overnight, processed routinely, and stained with haematoxylin and eosin at three levels before being examined by light microscopy. 
Table 1 Symptom complex at presentation of 50 women with chronic vulval complaints

\begin{tabular}{lc}
\hline Symptom complex & No of patients \\
\hline Pruritus vulvae only & 9 \\
Dyspareunia only & 1 \\
Vulvodynia only & 1 \\
Pruritus vulvae and dyspareunia & 10 \\
Pruritus vulvae and vulvodynia & 7 \\
Dyspareunia and vulvodynia & 8 \\
All three & 14 \\
\hline
\end{tabular}

\section{Results}

\section{SYMPTOMS}

The mean duration of symptoms was $3 \frac{1}{2}$ years, but ranged from two months to 27 years. Table 1 shows the symptom complex at presentation. Pruritus vulvae was a complaint of 40 patients, 33 had dyspareunia, and 30 had vulvodynia. A combination of two or more of these symptoms occurred in 39 of the women.

\section{MACROSCOPIC APPEARANCES}

Careful inspection showed one or more macroscopic abnormality in 29 of the women. Table 2 shows that the most common feature was erythema, which was noted in 14 women and in nine was confined to the posterolateral region of the vestibule. Thirteen women had papular or plaque like lesions that were associated usually with lichenification or an eczematous appearance, and a proportion were pigmented. These changes were located in the hair bearing areas of the vulva or perianally. Twelve of the women had fissures, which were located on the mucosal surfaces of six and in the remainder were associated with plaques. Overall, 18 patients had easily visible changes located in the hair bearing areas, and in two women these extended to include the labia minora. Eleven patients, however, had small fissures or erythema, or both, which were confined solely to the mucosal surface, usually at the fourchette and could easily have been overlooked without careful inspection in bright light.

COLPOSCOPIC APPEARANCES

Abnormalities were identified colposcopically in $\mathbf{4 5}$ of

Table 2 Macroscopic appearances on examination of 50 women with chronic vulval complaints

\begin{tabular}{ll}
\hline Appearance of lesion & $\begin{array}{l}\text { No of patients with } \\
\text { indicated lesion* }\end{array}$ \\
\hline Erythema & 14 \\
Papules or plaques & 13 \\
Fissuring & 12 \\
Ulceration & 4 \\
Atrophic or white epithelium & 2 \\
\hline
\end{tabular}

*More than one lesion observed in some patients.
Table 3 Colposcopic appearances on examination of 50 women with chronic vulval complaints

\begin{tabular}{ll}
\hline Appearance of lesion & $\begin{array}{l}\text { No of patients with } \\
\text { indicated lesion* }\end{array}$ \\
\hline Aceto white epithelium & 43 \\
Flat & 40 \\
Papillary & 22 \\
Cobblestone & 12 \\
Fissuring & 3 \\
Small warts & 2 \\
Ulceration & 1 \\
Erythema & 1 \\
\hline
\end{tabular}

*More than one lesion observed in some patients.

the women. Table 3 shows that the most common finding was acetowhite epithelium, which was observed in 43. Many of these women had more than one of the following three distinct patterns of epithelium: flat, which occurred on otherwise normal non-raised skin, including mucosal and keratinised surfaces; papillary, which comprised small multiple villous projections; and cobblestone, which comprised broader processes, possibly of fused papillae, raised above the surface and arranged in a cobblestone manner. The latter two types were confined to the mucous membrane of the vestibule, mainly at the fourchette (figure).

Colposcopy also showed other lesions in seven patients (table 3). Genital herpes was diagnosed in one patient after herpes simplex virus had been isolated from two small ulcers located on the inner aspect of the labium minorum. In another patient, a small fissure was evident at the frenulum of the clitoris, the site of her soreness for one year. A pea sized nodule was palpable, the excision of which resulted in improvement of her symptoms. Histology showed fibrous tissue.

\section{HISTOPATHOLOGICAL APPEARANCES}

Thirty three patients willing to undergo biopsy had vulval skin taken from lesions seen macroscopically or colposcopically; histological evaluation showed that all samples had abnormal features (table 4). Vulval intraepithelial neoplasia (VIN) was identified in samples from seven patients, grade I in five cases and grade III in two; six of these seven lesions were visible to the naked eye. Koilocytosis was observed in samples from 27 of the 33 patients. In 14 of them additional abnormalities were present as follows: seven had associated VIN, five had inflammatory epithelial hyperplasia (IEH), and two had non-specific inflammation. Vulval dermatoses were identified in samples from 10 of the patients as follows: two had lichen sclerosis (LS) and eight had IEH. Six of the eight with IEH had koilocytes in the lesions, as mentioned previously, and one had associated non-specific inflammatory changes. 


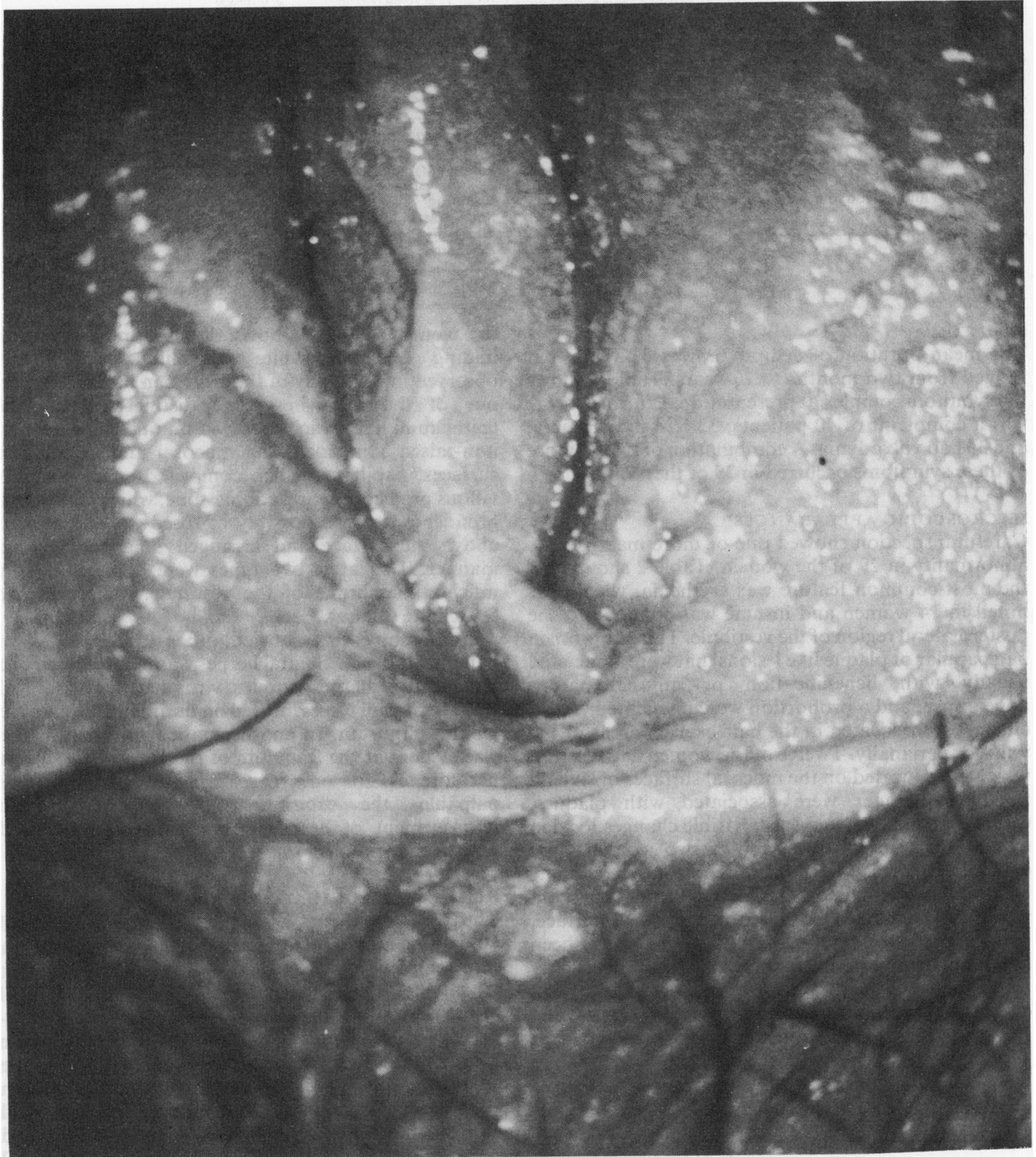

Figure Colpophotograph showing papillary and cobblestone patterns of acetowhite epithelium at the fourchette.

Cervical intraepithelial neoplasia (CIN) was detected in six women, all of whom had vulval koilocytosis. None of the women without such koilocytosis had CIN.
OTHER GENITAL TRACT INFECTIONS

All women were screened and treated for STD before their referral to the vulval clinic. Forty five had had one or more infections previously. Fifteen of the 27 
Table 4 Histopathological findings in 33 women with chronic vulval complaints

\begin{tabular}{llll}
\hline & \multicolumn{3}{l}{$\begin{array}{l}\text { No of patients who underwent } \\
\text { biopsies from lesions that were: }\end{array}$} \\
\cline { 2 - 4 } Histological finding & Macroscopic & Colposcopic & Total \\
\hline VIN & 4 & 1 & 5 \\
$\quad$ Grade I & 2 & 0 & 2 \\
$\quad$ Grade III & 6 & 7 & 13 \\
$\begin{array}{c}\text { Koilocytosis: } \\
\text { Alone }\end{array} \quad$ Combined & 10 & 4 & 14 \\
$\begin{array}{c}\text { Dermatoses: } \\
\text { Alone } \\
\text { Combined } \\
\text { Inflammatory changes: }\end{array} \quad 3$ & 0 & 3 \\
$\quad$ Alone & 4 & 3 & 7 \\
\hline
\end{tabular}

VIN $=$ Vulval intraepithelial neoplasia.

women with koilocytosis had had a previous STD, but only four had had classic genital warts and none of them had any that were clinically overt at the time of assessment. Six of the seven women with VIN had evidence of a previous STD.

\section{Discussion}

In this study women with unresolved chronic vulval symptoms were selected for detailed assessment that included a colposcopic examination of the vulva. A combination of symptoms was four times as prevalent as a single one. Although abnormalities were identified in the vulvae of all of them, naked eye inspection showed abnormal features in only 29 . Even so, this was the first time an abnormality had been documented in 16 patients, despite their previous examination in the routine clinic. Careful examination with a good light source is essential if abnormalities are to be detected, especially those that are minor or subtle in appearance. Colposcopy of the vulva was particularly rewarding in that various abnormalities were shown in 45 of the women. Indeed, without colposcopy lesions would not have been detected in 21 of them, and these women would have been said to have normal vulvae and would probably have been dismissed.

Most of the colposcopically detected abnormalities consisted of acetowhite epithelium, in the patterns described, confined to the mucosal surface. Human papillomavirus (HPV) has been reported in association with such changes. ${ }^{2}$ Further evidence suggestive of HPV infection was koilocytosis identified histologically in skin samples from 27 of the women who were biopsied. None of those women had overt classic warts at the time of assessment, and only four gave a history of having had them. The HPV infection was therefore either inapparent (in 16 patients) despite the presence of clinical lesions or was subclinical (in 11 patients) and shown only by colposcopy. This may represent an important, previously unrecognised, reservoir of virus. HPV has been implicated in the genesis of genital tract neoplasia, ${ }^{34}$ and in our study a quarter of the patients with koilocytosis had coexistent VIN or CIN, or both. Furthermore, VIN is associated commonly with other STD, ${ }^{5}$ and most of the women with VIN that we investigated had evidence of past STD. Of eight patients with IEH, six had associated koilocytosis. Whether these patients are at increased risk of developing neoplasia needs to be assessed by prospective follow up.

Vulval lesions may indicate disease elsewhere in the genital tract, and our observations showed that women with vulval disease that is associated with HPV infection may have an increased risk of cervical neoplasia. Thus CIN was detected in one fifth of the women with vulval koilocytosis, but in none of those without such koilocytosis. Examination of the mouth of one patient who had inflammatory vulval changes showed lesions typical of lichen planus, and this was identified subsequently in her vulva. This emphasises the importance of general examination of patients, particularly those whose vulval disease remains undiagnosed.

We conclude from this study that women with unresolved chronic vulval symptoms warrant detailed investigation because in many cases a firm diagnosis can be established and appropriate management instituted. These further investigations take time and require expertise in colposcopy. Many such women appear to be infected with HPV and are at increased risk of multicentric genital tract neoplasia, but further work is necessary to assess whether these viruses are a cause of neoplasia and whether they might be responsible for the chronic symptoms. Genitourinary physicians need to be familiar with the expanding clinical range of HPV infection and the increasing prevalence of VIN, as women with these problems may be expected to attend STD clinics in even larger numbers in the future.

We thank the consultants at the Praed Street Clinic for allowing us to study patients under their care and Mrs Kathy Jameson for typing the manuscript.

\section{References}

1 Burning vulva syndrome: report of the ISSVD task force. J Reprod Med 1984;29:457.

2 di Paola GR, Rueda NG. Deceptive vulvar papillomavirus infection. J Reprod Med 1986;31:966-70.

3 zur Hausen H, Gissman L, Schlehofer JR. Viruses in the etiology of human genital cancer. Prog Med Virol 1984;30:170-86.

4 Sutton GP, Stehman FB, Ehrlich CE, Roman A. Human papillomavirus deoxyribonucleic acid in lesions of the female genital tract: evidence for type 6/11 in squamous carcinoma of the vulva. Obstet Gynecol 1987;70:564-8.

5 Friedrich EG, Wilkinson EJ, Fu YS. Carcinoma in situ of the vulva: a continuing challenge. Am J Obstet Gynecol 1980:136:830-8. 